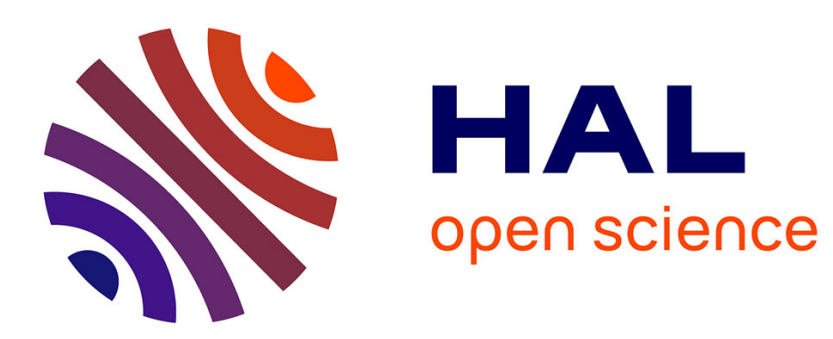

\title{
A comparison of two theories to explain the rotation curves in galaxies without dark matter particles
}

\author{
Barrie W Jervis
}

\section{To cite this version:}

Barrie W Jervis. A comparison of two theories to explain the rotation curves in galaxies without dark matter particles. Astrophysics and Space Science, 2018, 10.1007/s10509-018-3474-z . hal-01955413

\section{HAL Id: hal-01955413 \\ https://hal.science/hal-01955413}

Submitted on 14 Dec 2018

HAL is a multi-disciplinary open access archive for the deposit and dissemination of scientific research documents, whether they are published or not. The documents may come from teaching and research institutions in France or abroad, or from public or private research centers.
L'archive ouverte pluridisciplinaire HAL, est destinée au dépôt et à la diffusion de documents scientifiques de niveau recherche, publiés ou non, émanant des établissements d'enseignement et de recherche français ou étrangers, des laboratoires publics ou privés. 
"Towards Reconciling Gravitational Theories"

This paper has been thoroughly revised, and the title has been changed to:

A comparison of two theories to explain the rotation curves in galaxies without dark matter particles

This is a pre-print of an article published in Astrophysics and Space Science. The final authenticated version is available online at: https://doi.org/10.1007/s10509-

$\underline{018-3474-z}$

Reference: Astrophysics and Space Science, December 2018, 363:254

Running head: A Comparison of Two Theories

Type: Cosmology research article

Title: A Comparison of Two Theories to explain the Rotation Curves in Galaxies without Dark Matter Particles

Author: Barrie W. Jervis

Sheffield Hallam University, England, retired professor.

115 Button Hill, Sheffield, S11 9HG, England.

Tel: 44-114 2962170

barrie.jervis@cantab.net 


\section{Abstract}

The aim was to demonstrate the possible compatibility between the gravitational theory of Hajdukovic, which depends upon the existence of virtual particle pairs in vacuo, and that of emergent gravity according to Verlinde, and to derive new physical relationships. These theories, which avoid the necessity for postulating dark matter, and necessary, associated concepts are described. Then, assuming the validity of these theories, the following new results are obtained. Assuming a spherical galaxy model, the theories predict approximately the same radial distance from the galactic centre for the onset of enhanced gravitational fields. This agreement is used (1) to show that the virtual particle density of the vacuum at this distance depends only upon the Hubble scale and Planck's constant, (2) to calculate its value, and (3) to show that the particles consist of closely packed pions.

Keywords: emergent gravity, gravitational dipoles, spherical galaxy, dark matter

\section{Introduction}

Zwicky (1933) observed anomalous velocity dispersion in the Coma cluster of galaxies, and Rubin (1970) discovered that at large radii galactic material rotates faster than predicted by Newton's Laws. Both these observations suggested the presence of stronger than expected gravitational fields. The two most popular theories to explain these, and other galactic phenomena, are the presence of undiscovered matter (dark matter) or the Modified Newtonian Dynamics theory, MOND (Bekenstein and Milgrom, 1984), of the modification of the laws of gravity. Dark matter is postulated to consist of massive, weakly-interacting, cold, dark particles, which neither emit nor reflect light. The location of these particles, assuming they exist, has been determined by computer modelling. 
Recently two alternative, promising theories of gravity have been proposed which remain to be fully tested, but have explained some of the gravitational effects of galaxies without the need for dark matter. The theory of emergent gravity is based upon a melange of the theories of thermodynamics, entropy, black holes, information, elasticity, and string theory, as described in a very recent paper (Verlinde, 2017). Gravity is shown to emerge from the quantum bits of information which characterise space-time, and is, therefore, not a fundamental property of matter. According to the second theory (Hajdukovic, 2011), the additional gravitational force associated with galaxies is contributed by the partial alignment of gravitationally polarised virtual particle pairs in an external gravitational field. The pairs of particles and antiparticles spontaneously appear very briefly and then selfannihilate, according to Heisenberg's Uncertainty Principle and Einstein's equation of equivalence between mass and energy, $E=m c^{2}$. The antiparticles are assumed to have negative mass, as well as negative charge. This will be called the Hajdukovic theory, after its inventor. It is these two theories which are considered in this paper.

In this new work founded upon the assumed validity of these theories, it is shown that, based on a spherical galactic model, the two theories predict an enhanced gravitational effect at the same location. Then, by combining equivalent formulaic results from the two theories, it is shown that the virtual particle density in the galactic halo depends upon the Planck wavelength and the size of the universe. The particle density is calculated and the particles are shown to be pions.

Some underlying concepts and the two new theories are first reviewed. The new contribution appears in Sections 4 and 5. 


\section{Underlying Concepts}

\subsection{The Evidence for Dark matter}

Modelling shows that most galaxies are surrounded by a halo of dark matter. These haloes have a constant central surface density (Donato et al., 2009). Also studies of the dwarf galaxies Fornax and Sculptor show dark matter distributed uniformly within the central region ( Walker and Peñarrubia, 2007)]. Thus a uniform density is characteristic of dark matter, and there may be dark matter within a galaxy as well as surrounding it. Further evidence for possible dark matter is offered by the lensing effect of the Bullet Cluster of colliding galaxies (Clowe et al., 2008) and from the cosmic background microwave radiation (CMBR) (Einasto, 2011). However, the existence of dark matter has not been proved. Any new theory must account for these phenomena.

\subsection{Virtual Particle Pairs}

It is fundamental to Hajdukovic's theory that the vacuum of space is full of virtual particle-pairs. Their existence has been shown by the Casimir Effect (Casimir, 1948) and the Lamb shift (Lamb, 1947)..It has been suggested that gravitational polarisation of these particles accounts for the dark matter explained by the MOND model (Blanchet, 2007) as well as for dark matter and dark energy (Blanchet and Tiec, 2008, 2009). In Hajdukovic's theory these ideas are extended to the gravitational polarisation of the virtual dipoles.

\subsection{Entropy, Information, Black Holes, and Entropic Force}

These concepts are used in the theory of emergent gravity (Verlinde, 2017) and so relevant aspects are reviewed here. Thermodynamic entropy is given by the wellknown Boltzmann equation

$$
S=k_{B} \ln W
$$


in which $k_{B}$ is Boltzmann's constant, $W$ is the number of possible states, and $\ln$ denotes logarithm. In information theory information is conveyed in strings of $N$ binary bits. The information entropy, is given by

$$
S=\log _{2} 2^{N}
$$

These equations are identical in form, showing that thermodynamic entropy and information entropy are interchangeable.

Microscopically, in the domain of quantum mechanics, the binary information bits become qubits taking any value between 0 and 1 . When the quantum mechanical wave function represents two states simultaneously the two states are said to be entangled. Thus, their representative qubits are also entangled. In the theory of black holes (Bekenstein, 1973) the entropy of a black hole may be regarded as the number of associated entangled qubits.

An increase in entropy, $\Delta S$, is associated with the work done, $F \Delta x$, by the application of a force, $F$, in moving an object through a distance $\Delta x$. The energy increase in entropy terms is $T \Delta S$, where $T$ is the temperature. Equating these gives

$$
T \Delta S=F \Delta x
$$

\section{The New Gravitational Theories}

\subsection{Theory of Emergent Gravity}

Verlinde presented a theory of emergent gravity (Verlinde, 2017), and a nonmathematical description has been given by Visser and Vonk, (2017). According to Verlinde an empty space is filled with dark energy, which provides a volume contribution to the total entropy. It also acts as an expansive force, which drives the expansion of the universe. If some of this energy is converted into ordinary baryonic 
matter, the entropy change produces a force, according to equation (3). The universe is considered an elastic medium and this force results in elastic strain, which slowly relaxes over a very long timescale. At the microscopic quantum scale, black hole theory is applied, and the entropy is associated with qubits. The creation of matter displaces the qubits, leaving a vacuum in the vicinity of the mass. The qubits push to fill the vacuum, which creates an additional gravitational force. This phenomenon is significant whenever the mass is sufficiently large, and causes the large gravitational fields in the vicinity of galaxies. Thus, gravity is regarded as an emergent macro property of the microscopic behaviour of the qubits.

Verlinde has demonstrated how Newton's Laws may be derived from this theory (Verlinde, 2011).

\subsection{Hajdukovic's Theory}

Hajdukovic produced his theory to explain the phenomenon of dark matter (Hajdukovic, 2011). The virtual particles and antiparticles are assumed to have opposite gravitational charges and to form gravitational dipoles and opposite gravitational charges are assumed to repel each other. The concept of matter and antimatter being mutually repulsive has been investigated theoretically (Villata, 2011) with the conclusion that antigravity is predicted by the theory of general relativity. See also (Hajdukovic, 2009)._Experiments to detect negative mass by observing in which direction neutral antihydrogen atoms fall are being undertaken at CERN in the AEGIS, ALPHA, and GBAR experiments. Hajdukovic also assumes that the virtual particle pairs constitute a gas of virtual pions, which are quark-antiquark pairs (Hajdukovic, 2010). Pions may have the smallest mass possible in nature (Böhmer and Harko, 2005), and Hajdukovic argues that pions may dominate the content of 
the quantum vacuum (Hajdukovic, 2010). The separation between the poles of the opposite gravitational charges of the virtual dipoles $\cong \lambda_{c}=h / m c$, their Compton wavelength, at their equilibrium separation. Here $c$ is the velocity of light, $h$ is the Planck constant, and $m$ is the mass of the pion. Further, according to quantum field theory, a virtual particle pair should occupy the volume $\lambda_{c}^{3}$ (Hajdukovic, 2011). Thus, the particle pair density in the vacuum is $N=1 / \lambda_{c}^{3}$.

These polarised particle pairs become aligned in a gravitational field to an extent which depends upon the field strength, just as electric dipoles align in an electric field. Hajdukovic used a simple spherical model of a galaxy and calculated the radial gravitational charge density. The evidence for a uniform matter density in regions of dark matter has already been mentioned above (Donato et al, 2009), (Walker and Peñarrubia, 2011), and so regions of uniform gravitational charge density were taken to represent dark matter regions. In fact the radial distance from the centre at which the dark matter effect was to be found was determined to be

$$
R_{0}=\lambda_{c} \sqrt{\frac{M_{b}}{m_{c}}}
$$

in which $M_{b}$ is the baryonic mass. Hajdukovic took $\lambda_{c}=\lambda_{\pi}$ and $m_{c}=m_{\pi}$ because he was considering pions.

\subsection{Gravitational Theories - the Evidence}

Table 1 gives a summary of the successes, $\sqrt{ }$, failures, $X$, and unknown predictions,?, for the supposed dark matter phenomena, as known by the author at present, by the different theories in different instances.

Table 1 
The MOND theory predicted that the Milky Way would exhibit a disc of dark matter, while Hajdukovic's theory did not predict one. Attempts to find it were unsuccessful (Bidin et al., 2010). The emergent gravity theory has been successfully tested for the case of gravitational lensing (Brewer et al., 2017). While much more work remains to be done, the table shows that the emergent gravity and Hajdukovic's theories look promising, whilst the MOND theory failed in two instances.

\section{Comparing the two Theories}

As Verlinde's theory of emergent gravity and Hajdukovic's theory both explain galactic haloes and gravitational lensing, they should predict the same radial distance from the centres of spherical galaxies to the onset of dark matter effects. This distance was given in equation (4) for Hajdukovic's theory. Let us now apply the theory to the spherical galaxy Messier 89 (NGC4552). Its mass is $3.2 \times 10^{42} \mathrm{~kg}$ for a half-light radius of $1.5 \times 10^{21} \mathrm{~m}$. Substituting $h / m c$ for $\lambda_{c}$, putting $m=2.4 \times 10^{-28} \mathrm{~kg}$ as the mass of a pion, and using $M_{b}=3.2 \times 10^{42} \mathrm{~kg}$, gives $R_{0}=1.06 \times 10^{21} \mathrm{~m}$. This radius is a little less than the half-light radius, and suggests that some dark matter is present within the outer layers of the galaxy.

For emergent gravity, with $A$ as the surface area of a sphere of radius $R$ surrounding the galactic centre, beyond which dark matter is predicted, Verlinde's equation (4) in (Verlinde, 2017) describes the situation by,

$$
\frac{A}{4 G \hbar}>\frac{2 \pi M}{\hbar a_{0}}
$$

where $M$ is the enclosed mass, $G$ is the gravitational constant, and $a_{0}$ is the cosmological acceleration scale, given by

$$
a_{0}=\frac{c^{2}}{L}
$$

where $L$ is the Hubble scale, the radius of the universe. Upon substituting equation (6) into equation (5), putting $A=4 \pi R^{2}$, and rearranging gives 


$$
R>\sqrt{2 M L G} / c
$$

Again, taking the case of the spherical galaxy, Messier $89, M=3.2 \times 10^{42} \mathrm{~kg}$, while $L=4.4 \times 10^{25} \mathrm{~m}, G=6.67 \times 10^{-11} \mathrm{~m}^{3} \mathrm{~kg}^{-1}$, and $c=3 \times 10^{8} \mathrm{~m} \mathrm{~s}^{-1}$ giving $R>4.57 \times$ $10^{20} \mathrm{~m}$. Again this distance is less than the half-light radius, suggesting the presence

of dark matter within the outer layers of the galaxy. Taking the ratio, $R_{0} / R=2.32$.

Considering that the two theories were derived from different models, this is considered to be a good agreement. See also Section 6.

\section{Combining the two theories}

The evidence in Table 1, and the agreement between $R$ and $R_{0}$, suggest the emergent gravity and Hajdukovic theories are compatible. Then, since equations (4) and (7) give essentially the same result, they may be equated to give the new relationship

$$
\lambda_{c} \sqrt{\frac{M}{m_{c}}}=\sqrt{2 M L G} / c
$$

This new equation may be applied to derive new results as follows. Substituting $m_{c}=h / \lambda_{c} c$ and $N=1 / \lambda_{c}^{3}$, it is found that

$$
N L=\frac{c^{3}}{2 G h}
$$

showing that the particle density at $R_{0}$ only depends upon known constants and the radius of the spherical universe. The Planck wavelength, $\lambda_{P}$, is

$$
\lambda_{P}=\sqrt{\frac{\hbar G}{c^{3}}}
$$

And so combining equations (9) and (10) gives 


$$
N L=\frac{1}{4 \pi \lambda_{P}^{2}}
$$

We can define an area proportional to $\lambda_{P}^{2}$ as the Planck area showing that $N L$ is inversely proportional to the smallest meaningful area of space. The particle density at $R_{0}$ can be estimated from equation (9) as

$$
N=\frac{c^{3}}{2 G h L}
$$

Substituting values gives $N=2.31 \times 10^{42}$ particles $\mathrm{m}^{-3}$. This value compares well with the value calculated directly from $N=1 / \lambda_{c}^{3}$, which is $1.28 \times 10^{42} \mathrm{~m}^{-3}$.

So far the type of particle has not been defined. If we assume, like Hajdukovic [5], that they are close neighbours with $N=1 / \lambda_{c}^{3}$ then $\lambda_{c}=N^{-1 / 3}=7.56 \times 10^{-15} \mathrm{~m}$. This is comparable to $\lambda_{c}$ for the pion $\left(9.20 \times 10^{-15} \mathrm{~m}\right)$ and it is thought that the quantum vacuum is dominated by pions (Hajdukovic, 2011). This approximate agreement is further evidence that the two theories yield similar results and that equation (8) is valid. The physical meaningfulness of equation (11) also suggests the compatibility of the two theories.

In deriving the expression for $R_{0}$, equation (4), the spacing between the virtual particle and its antiparticle was taken to be approximately $\lambda_{c}$ according to quantum field theory, and the virtual dipole pairs were assumed tightly packed so that the particle density is $N=1 / \lambda_{c}^{3}$. This second assumption can now be tested. Let the particle density be $N=1 / x^{3}$ where $x \geq \lambda_{c}$. Then from equation (8)

$$
x=\frac{\sqrt{2 L G m_{c}}}{c}
$$


if pions are assumed, $m_{c}=2.4 \times 10^{-28} \mathrm{~kg}$, whence $x=3.2 \times 10^{-15} \mathrm{~m}$, compared with $\lambda_{\pi}=9.203 \times 10^{-15} \mathrm{~m}$. These values agree within the factor of 2.9, suggesting the pions are indeed closely packed. This is more evidence for the compatibility of the two theories. Note that $R_{0} / R=\frac{\lambda_{\pi}}{x}=h / \sqrt{2 m_{\pi}^{3} L G}=2.33$. If the theories were exact, this could infer that $L$ has been underestimated by the factor $2.33^{2}=5.43$. If this is not the case, then the theories yield very close, rather than exactly the same values.

\section{Conclusion}

The predicted agreements with the "observations" of dark matter, and the calculated approximate agreement shown here between the predicted radial locations of the supposed dark matter for a spherical galactic model suggest the theories of Hajdukovic and of emergent gravity are comparable. Assuming the validity of these theories and for the first time equating the two formulae for the radial location of the dark matter effect, it is shown that the virtual particle density in this region depends only upon the Hubble scale and the Planck area, $\propto \lambda_{P}^{2}$. This new basic relationship is physically credible and also suggests compatibility between the two theories. Using this new relationship the number density of virtual particle pairs was calculated to be $2.31 \times 10^{42} \mathrm{~m}^{-3}$, and their separation by approximately one Compton wavelength corresponded to that of tightly packed pions.

\section{Acknowledgements}

The author thanks Dr A. J. Jervis for providing some references and comments on the manuscript, and John Edwards and David Barber for feedback.

\section{References}

Zwicky, F., 1933, Helvetica Physica Acta, 6, 110 
DOI http://adsabs.harvard.edu/abs/1933AcHPh...6..110Z

Rubin, V., \& Ford, W. K., 1970, The Astrophysical Journal, 159, 379

DOI 379 10.1086/150317.

Bekenstein, J., \& Milgrom, M., 1984, Astrophysical Journal, 286, 7

DOI $10.1086 / 162570$

Verlinde, E. P., 2017, SciPost Phys.

DOI10.21468/SciPostPhys.2.3.016

Hajdukovic, D. S, 2011, Astrophys Space Sci, 334, 215

DOI 10.1007/s10509-011-0744-4

Donato, F., Gentile, G., Salucci, P., Martins, F., Wilkinson, M. I., Gilmore, G., Grebel, E.K., Koch, A., \& Wyse, R., 2009, Mon. Not. R. Astron. Soc., 397, 1169

DOI 10.1111/j.1365-2966.2009.15004.x

Walker, M., and Peñarrubia, J., 2011, The Astrophysical Journal, 742, 1

DOI10.1088/0004-637X/742/1/20

Clowe, D., Bradač, M., Gonzalez, A. H., Markevitch, M., Randall, S. W., Jones, C.,

\& Zaritsky, D., 2006, The Astrophysical Journal Letters, 648, 109

DOI10.1086/508162

Einasto, J., 2011, Baltic Astronomy, 20, 231

DOI 10.1515/astro-2017-0287

Casimir, H. B. G., 1948, Proc. Kon. Ned. Akad. Wetensch, B51, 793

Lamb, W. E., \& Retherford, R. C., 1947, Physical Review, 72, 241

DOI 10.1103/PhysRev.72.241.

Blanchet, L., 2007, Class. and Quant. Grav., 24, 3

DOI 10.1088/0264-9381/24/14/001

Blanchet, L., \& Tiec, A., 2008, Phys. Rev., D78, 024031 
DOI 10.1103/PhysRevD.78.024031

Blanchet, L., \& Tiec, A., 2009, Phys. Rev., D80, 023524

DOI 10.1103/PhysRevD.80.023524

Bekenstein, J. D., 1973, Phys. Rev., D 7, 2333

DOI 10.1103/PhysRevD.7.2333

Visser M., \& Vonk, M., 2017, at Quantum Universe website, 20/02/2017, 2305

Verlinde, E., 2011, Journal High Energy Physics, 29, 1

DOI10.1007/JHEP04(2011)029, ArXiv ePrint:1001.0785

Villata, M., Europhysics Letters, 94, 20001

DOI 10.1209/0295-5075/94/20001

D. Hajdukovic, D., 2009 at arXiv:0810.3435v3 [physics.gen-ph]

Hajdukovic, D. S., 2010, Europhysics Letters, 89, 1

DOI 10.1209/0295-5075/89/49001

Böhmer, C. G., \& Harko, T., 2005, Physics Letters B, 630, 73

DOI10.1016/j.physletb.2005.09.071

Bidin, C. M., Carraro G., Méndez, R. A., \& van Altena, W. F., 2010, The

Astrophysical Journal Letters, 724, 122

DOI 10.1088/2041-8205/724/1/L122

Brouwer, M. M., Visser, M. R., Dvornik, A., Hoekstra, H., Kuijken, K., Valentijn, E. A., Bilicki, M., Blake, C., Brough, S., Buddelmeijer, H., Erben, T., Heymans, C., Hildebrandt, H., Holwerda, B. W., Hopkins, A. M., Klaes, D., Liske, J., Loveday, J., McFarland, J., Nakajima, R., Sifón, C., \& Taylor, E. N., 2017, Monthly Notices of the Royal Astronomical Society, 466, 2547

DOI 10.1093/mnras/stw3192 
Table 1 The theoretical predictions compared to the locations of known dark matter

\begin{tabular}{|l|c|c|c|}
\hline \multicolumn{1}{|c|}{$\begin{array}{c}\text { Dark matter } \\
\text { phenomenon }\end{array}$} & MOND & Emergent gravity & $\begin{array}{c}\text { Gravitational } \\
\text { dipoles }\end{array}$ \\
\hline Galactic haloes & $\sqrt{ }$ & $\sqrt{ }$ \\
\hline Dwarf galaxies & $\sqrt{ }$ & $?$ \\
\hline $\begin{array}{l}\text { Gravitational } \\
\text { lensing }\end{array}$ & $\mathrm{X}$ & $\sqrt{ }$ \\
\hline Milky Way disc & $\mathrm{X}$ & $?$ & $\sqrt{ }$ \\
\hline
\end{tabular}

